\title{
First optical light from the supernova remnant G 17.4-2.3
}

\author{
P. Boumis ${ }^{1}$, F. Mavromatakis ${ }^{1}$, and E. V. Paleologou ${ }^{2}$ \\ 1 University of Crete, Physics Department, PO Box 2208, 71003 Heraklion, Crete, Greece \\ 2 Foundation for Research and Technology-Hellas, PO Box 1527, 71110 Heraklion, Crete, Greece
}

Received 19 November 2001 / Accepted 29 January 2002

\begin{abstract}
Deep optical CCD images of the supernova remnant G 17.4-2.3 were obtained and faint emission has been discovered therein. The images, taken in the emission lines of $\mathrm{H} \alpha+[\mathrm{NII}]$, [S II] and [O III], reveal filamentary structures in the east, south-east area, while diffuse emission in the south and central regions of the remnant is also present. The radio emission in the same area is found to be well correlated with the brightest optical filament. The flux calibrated images suggest that the optical filamentary emission originates from shockheated gas $([\mathrm{S} \mathrm{II}] / \mathrm{H} \alpha>0.4)$, while the diffuse emission seems to originate from an $\mathrm{H}$ II region $([\mathrm{S} \mathrm{II}] / \mathrm{H} \alpha<0.3)$. Furthermore, deep long-slit spectra were taken at the bright [O III] filament and clearly show that the emission originates from shock heated gas. The [O III] flux suggests shock velocities into the interstellar "clouds" greater than $100 \mathrm{~km} \mathrm{~s}^{-1}$, while the $[\mathrm{S} \mathrm{II}] \lambda 6716 / 6731$ ratio indicates electron densities $\sim 240 \mathrm{~cm}^{-3}$. Finally, the H $\alpha$ emission has been measured to be between 7 to $20 \times 10^{-17} \mathrm{erg} \mathrm{s}^{-1} \mathrm{~cm}^{-2} \operatorname{arcsec}^{-2}$.
\end{abstract}

Key words. ISM: general - ISM: supernova remnants - ISM: individual objects: G 17.4-2.3

\section{Introduction}

The Galactic supernova remnants (SNRs) have been identified by both radio (non-thermal synchrotron emission) and optical (optical emission lines) surveys. New searches in both wavebands continue to identify galactic SNRs (Fesen \& Hurford 1995; Fesen et al. 1997; Green 2000 and references therein; Mavromatakis et al. 2000, 2001, 2002) but since the last few years, observations in X-rays have also detected new SNRs (e.g. Seward et al. 1995). The ratio of $[\mathrm{S} \mathrm{II}] / \mathrm{H} \alpha$ has become the standard discriminator used in optical SNR observations because the photoionized nebulae (like $\mathrm{H}$ II regions and planetary nebulae) usually exhibit ratios of about $0.1-0.3$, while collisionally ionized nebulae (like known Galactic SNRs) show ratios typically greater than 0.4 (Smith et al. 1993). Fesen et al. (1985) suggested that a division at $[\mathrm{S} \mathrm{II}] / \mathrm{H} \alpha<0.5$ does not provide clear evidence to distinguish SNRs from photoionized regions and additional observations of the strong forbidden oxygen lines ([O I $],\left[\mathrm{O}_{\mathrm{II}}\right]$ and $[\mathrm{O} \mathrm{III}]$ ) are needed to give a complete diagnostic. Furthermore, theoretical shock models, generally predicted $[\mathrm{S}$ II $] / \mathrm{H} \alpha$ ratios of 0.5 to 1.0 for SNRs (Raymond 1979; Shull \& McKee 1979).

G 17.4-2.3 is not a well known SNR, and was first detected by Reich et al. (1988) in their Effelsberg 2.7-GHz

Send offprint requests to: P. Boumis, e-mail: ptb@physics.uoc.gr survey, while its radio image was published by Reich et al. (1990). It is classified as a circular supernova remnant with an incomplete radio shell, characterized by diffuse shell-like emission, an angular size of $\sim 24^{\prime}$ and a radio spectral index of $\sim 0.8$ (Green 2000). Case \& Bhattacharya (1998) calculated its surface brightness to be $1.3 \times 10^{-21} \mathrm{~W} \mathrm{~m}^{-2} \mathrm{~Hz}^{-1} \mathrm{~m}^{-1}$. Because, there is no direct distance determination, they have made an estimation by utilizing the radio surface brightness - diameter relationship $(\Sigma-D)$ and found a distance of $8.5 \mathrm{kpc}$, but still the uncertainties are large $(\sim 40 \%)$. Green et al. (1997), through their survey with the Parkes $64 \mathrm{~m}$ telescope, detected maser $\mathrm{OH}(1720 \mathrm{MHz})$ emission. In radio surveys of the surrounding region, no pulsar was found to be associated with G 17.4-2.3 but another SNR has been discovered in its neighborhood. G $17.8-2.6$ has a very well-defined shell, it lies about $30^{\prime}$ north-east of G 17.4-2.3 and has an angular diameter of $24^{\prime}$ (Reich et al. 1988). Neither of these remnants has been detected optically in the past and from our observations no optical emission has been found in $\mathrm{G}$ 17.8-2.6. On the other hand, $\mathrm{X}$-ray emission was not detected from G 17.4-2.3 in the ROSAT All-sky survey, while there is some evidence of $\mathrm{X}$-ray emission from the neighboring SNR G 17.8-2.6.

In this paper, we report the discovery of faint optical filaments from G 17.4-2.3. We present $\mathrm{H} \alpha+[\mathrm{NII}]$, $[\mathrm{S} \mathrm{II}]$ and $[\mathrm{O} \mathrm{III}]$ images which show filamentary structure along the south-east edge of the remnant correlated very 
well with the radio emission. Spectrophotometric observations of the brightest filament were also obtained and the emission lines were measured. In Sect. 2, we present informations concerning the observations and data reduction, while the results of our imaging and spectral observations are given in Sects. 3 and 4, respectively. In the last section (Sect. 5) we discuss the physical properties of G 17.4-2.3.

\section{Observations}

\subsection{Imagery}

The observations presented here were performed with the $0.3 \mathrm{~m}$ Schmidt-Cassegrain (f/3.2) telescope at Skinakas Observatory in Crete, Greece in August 20 and 21, 2001. The $1024 \times 1024$ (with $19 \times 19 \mu \mathrm{m}^{2}$ pixels) Tektronix CCD camera was used resulting in a scale of $4^{\prime \prime} .1$ pixel $^{-1}$ and a field of view of $70^{\prime} \times 70^{\prime}$.

Two exposures in [O III] and [S II] of $2400 \mathrm{~s}$ each were taken during the observations, while one exposure of $1800 \mathrm{~s}$ and one of $2400 \mathrm{~s}$ were obtained with the $\mathrm{H} \alpha+[\mathrm{N} \mathrm{II}]$ filter. Note that the final images in each filter are the average of the individual frames.

The image reduction (bias subtraction, flat-field correction) was carried out using the standard IRAF and MIDAS and their negative gray-scale representation using the STARLINK Kappa and Figaro packages. The astrometry information was calculated for each image individually using stars from the Hubble Space Telescope (HST) Guide Star Catalogue (Lasker et al. 1999). The spectrophotometric standard stars HR 5501, HR 7596, HR 7950, and HR 8634 (Hamuy et al. 1992, 1994) were used for absolute flux calibration. All coordinates quoted in this paper refer to epoch 2000 .

\subsection{Spectroscopy}

Low dispersion long-slit spectra were obtained with the $1.3 \mathrm{~m}$ telescope at Skinakas Observatory in 2001 August 21 . The 1300 line $\mathrm{mm}^{-1}$ grating was used in conjunction with a $2000 \times 800$ SITe CCD $\left(15 \times 15 \mu \mathrm{m}^{2}\right.$ pixels $)$ which resulted in a scale of $1 \AA$ pixel $^{-1}$ and covers the range of $4750 \AA-6815 \AA$. The slit width is $7^{\prime \prime} .7$ and it was oriented in the south-north direction, while the slit length is $7 ! 9$. The coordinates of the slit centre are $\alpha=18^{\mathrm{h}} 31^{\mathrm{m}} 30^{\mathrm{s}}$ and $\delta=-14^{\circ} 51^{\prime} 33^{\prime \prime}$ and two spectra of 3600 s each were obtained. The spectrophotometric standard stars HR5501, HR7596, HR9087, HR718, and HR7950 were observed in order to calibrate the spectra of G 17.4-2.3.

\section{Imaging of G $17.4-2.3$}

\subsection{The $H \alpha+[N \|]$ and [S II] emission line images}

The major characteristic revealed from our $\mathrm{H} \alpha+\left[\mathrm{NII}_{\mathrm{II}}\right.$ and $[\mathrm{S}$ II] images (Figs. 1 and 2, respectively) seems to be the low surface brightness of G 17.4-2.3. The weak diffuse emission is present in the south, south-east and central
Table 1. Typically measured fluxes in the area of the brightest filament.

\begin{tabular}{lllll}
\hline \hline & $\mathrm{N}^{1}$ & $\mathrm{SW}^{1}$ & Center $^{1}$ & $\mathrm{H} \mathrm{II}^{2}$ \\
\hline $\mathrm{H} \alpha+[\mathrm{N} \mathrm{II}]$ & 116.0 & 111.2 & 108.0 & 99.7 \\
\hline$[\mathrm{S} \mathrm{II}]$ & 16.3 & 22.1 & 17.3 & 8.6 \\
\hline$[\mathrm{O}$ III $]$ & 13.0 & 4.7 & 22.1 & - \\
\hline
\end{tabular}

Fluxes in units of $10^{-17} \mathrm{erg} \mathrm{s}^{-1} \mathrm{~cm}^{-2} \operatorname{arcsec}^{-2}$.

${ }^{1}$ Median values over a $36^{\prime \prime} \times 21^{\prime \prime}$ box.

${ }^{2}$ Median values over a $60^{\prime \prime} \times 60^{\prime \prime}$ box.

areas of the remnant, while no emission is detected in the north-west part. The most interesting region lies in the east part of the remnant, where a filamentary structure exists, which is very well correlated with the radio emission. In Table 1, we present typical average fluxes measured in several locations within the field of G 17.4-2.3 including the unknown $\mathrm{H}$ in region which is located south-east of the remnant. A deeper study of these images shows that the emission from the brightest part of the remnant (east filament) originates from shock heated gas since we estimate a ratio $[\mathrm{S} \mathrm{II}] / \mathrm{H} \alpha \sim 0.4-0.6$, while a photoionization mechanism produces the south-east $\mathrm{H}$ II region ([S II $] / \mathrm{H} \alpha \sim 0.2-$ $0.3)$. The possibility that the H II emission contaminates the remnant's emission can not be ruled out since for some of the areas close to the filament as well as to the central region of the remnant, we estimate $[\mathrm{S} \mathrm{II}] / \mathrm{H} \alpha \sim 0.2-0.3$. The known dark, extended nebula LDN 379 (Lynds 1965), which is at a distance of $\sim 200 \mathrm{pc}$ (Hilton et al. 1986) is also visible in the low ionization images.

\subsection{The $[O \mathrm{III}] 5007 \AA$ A image}

In contrast to the previous results, the image of the medium ionization [O III] line shows clearly filamentary nature of the observed emission (Fig. 3). This bright filament extends for $\sim 24^{\prime}$ in the east, south-east, while no significant emission was found in other areas of the remnant. We do not detect [O III] emission where the diffuse $\mathrm{H} \alpha+[\mathrm{N}$ II $]$ and $[\mathrm{S} \mathrm{II}]$ emission is detected. Table 1 lists also typical [O III] fluxes measured in different parts of the filament. The latter, matches very well with the radio maps of G $17.4-2.3$ at $1400 \mathrm{MHz}$ and $4850 \mathrm{MHz}$, suggesting their association (Fig. 4). The correlation shows that the filament is located close to the outer edge of the radio contours but the low resolution of the radio images does not help us to determine the actual position of the filament with respect to the shock front. The geometry of the [O III] filament allows us to define a $\sim 26^{\prime}$ circle in diameter (dashed circle in Fig. 3) for the remnant, with its center at $\alpha \simeq 18^{\mathrm{h}} 30^{\mathrm{m}} 23.3^{\mathrm{s}}, \delta \simeq-14^{\circ} 45^{\prime} 25^{\prime \prime}$. Note that the optical angular size is in very good agreement with the value of $\sim 24^{\prime}$ given in Green's catalogue (Green 2000). However, a larger angular diameter cannot be excluded since X-ray emission has not been detected so far and the radio shell is incomplete. 


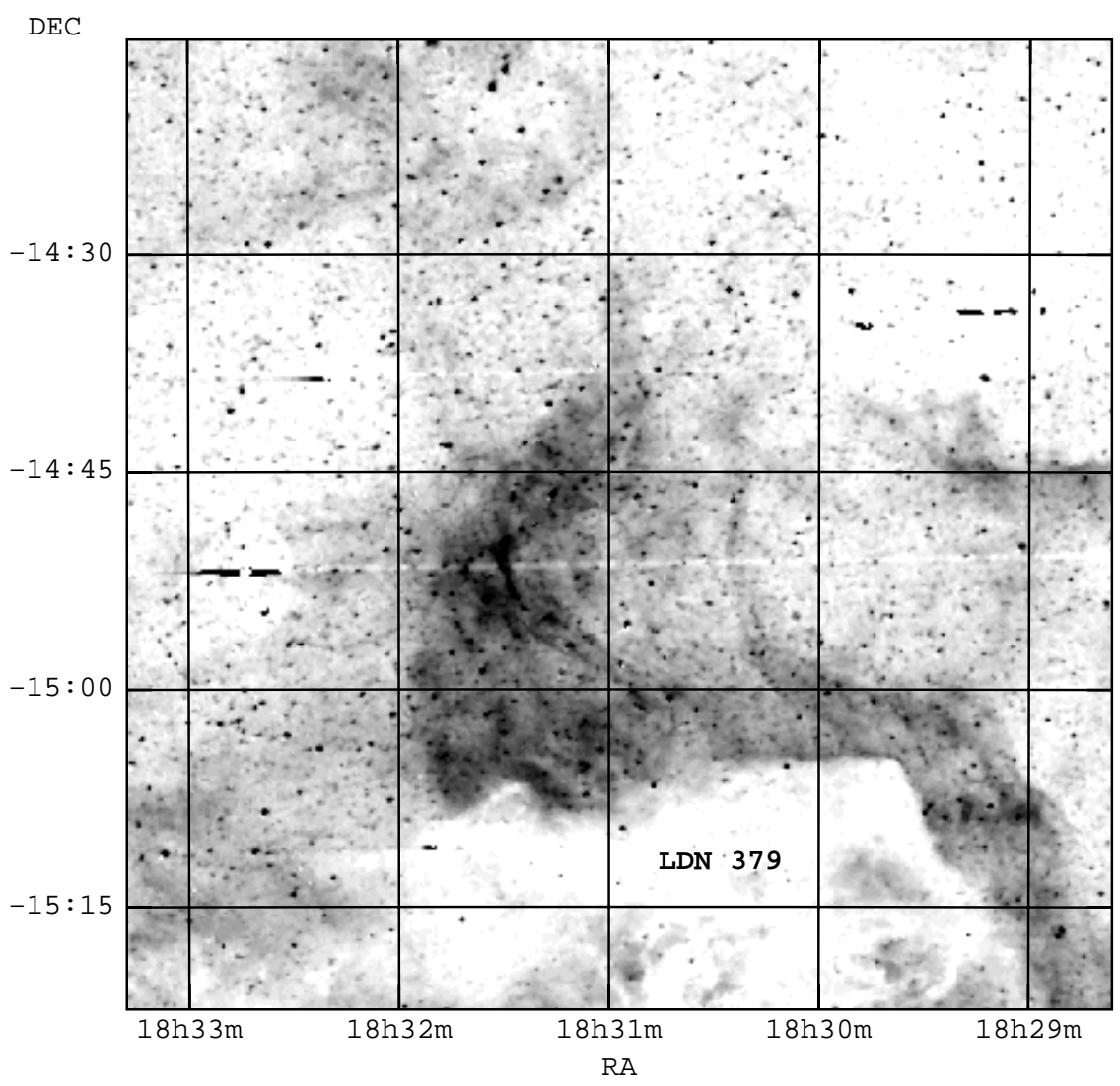

Fig. 1. The field of $\mathrm{G} 17.4-2.3$ in the $\mathrm{H} \alpha+[\mathrm{N}$ II] filter. The image has been smoothed to suppress the residuals from the imperfect continuum subtraction. Shadings run linearly from 0 to $120 \times 10^{-17} \mathrm{erg} \mathrm{s}^{-1} \mathrm{~cm}^{-2} \operatorname{arcsec}^{-2}$. The line segments seen near overexposed stars in this figure and the next figures are due to the blooming effect.

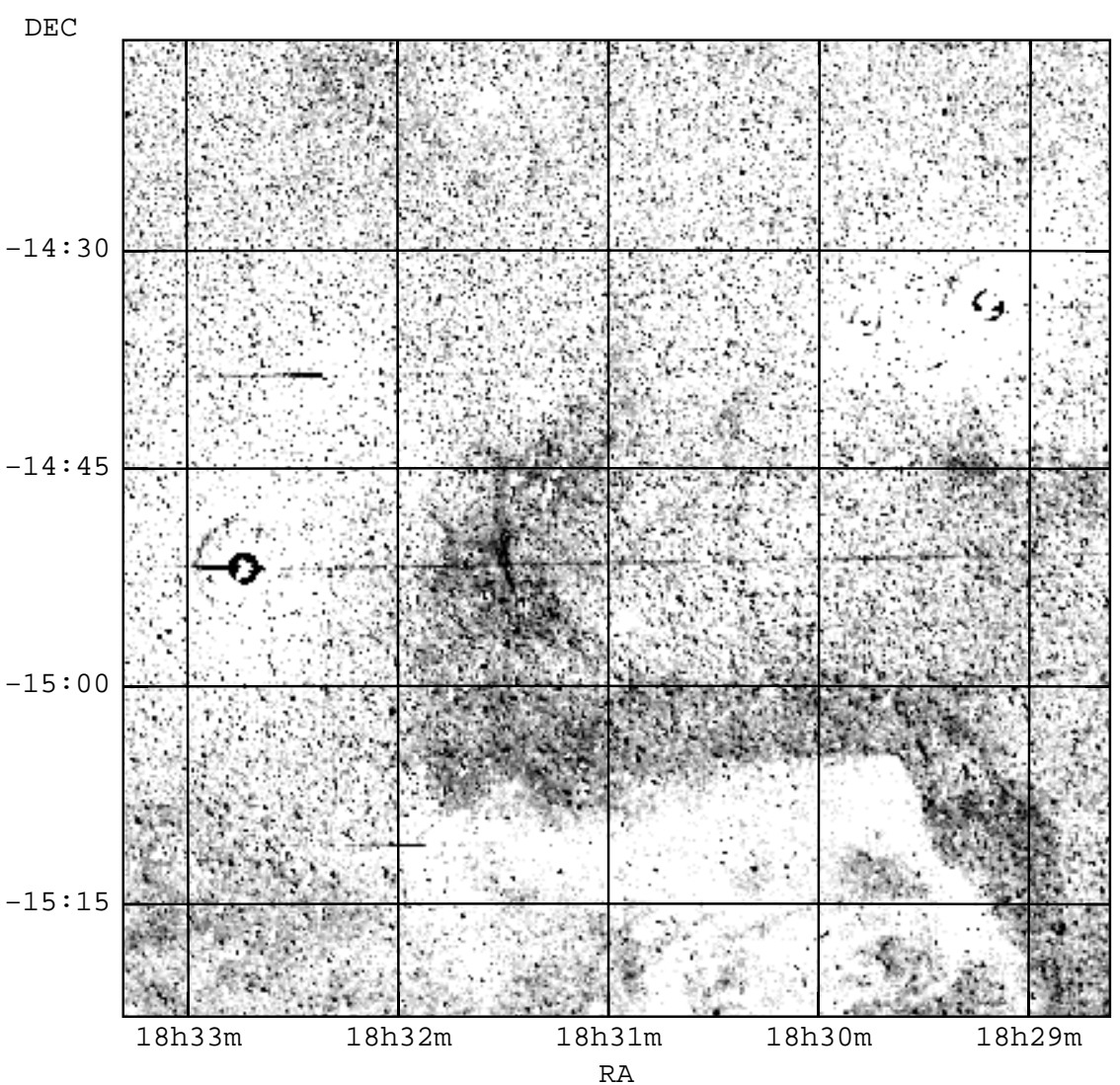

Fig. 2. The $[\mathrm{S}$ II $]$ image of the area around G 17.4-2.3, which has been smoothed to suppress the residuals from the imperfect continuum subtraction. Shadings run linearly from 0 to $20 \times 10^{-17} \mathrm{erg} \mathrm{s}^{-1} \mathrm{~cm}^{-2} \operatorname{arcsec}^{-2}$. 


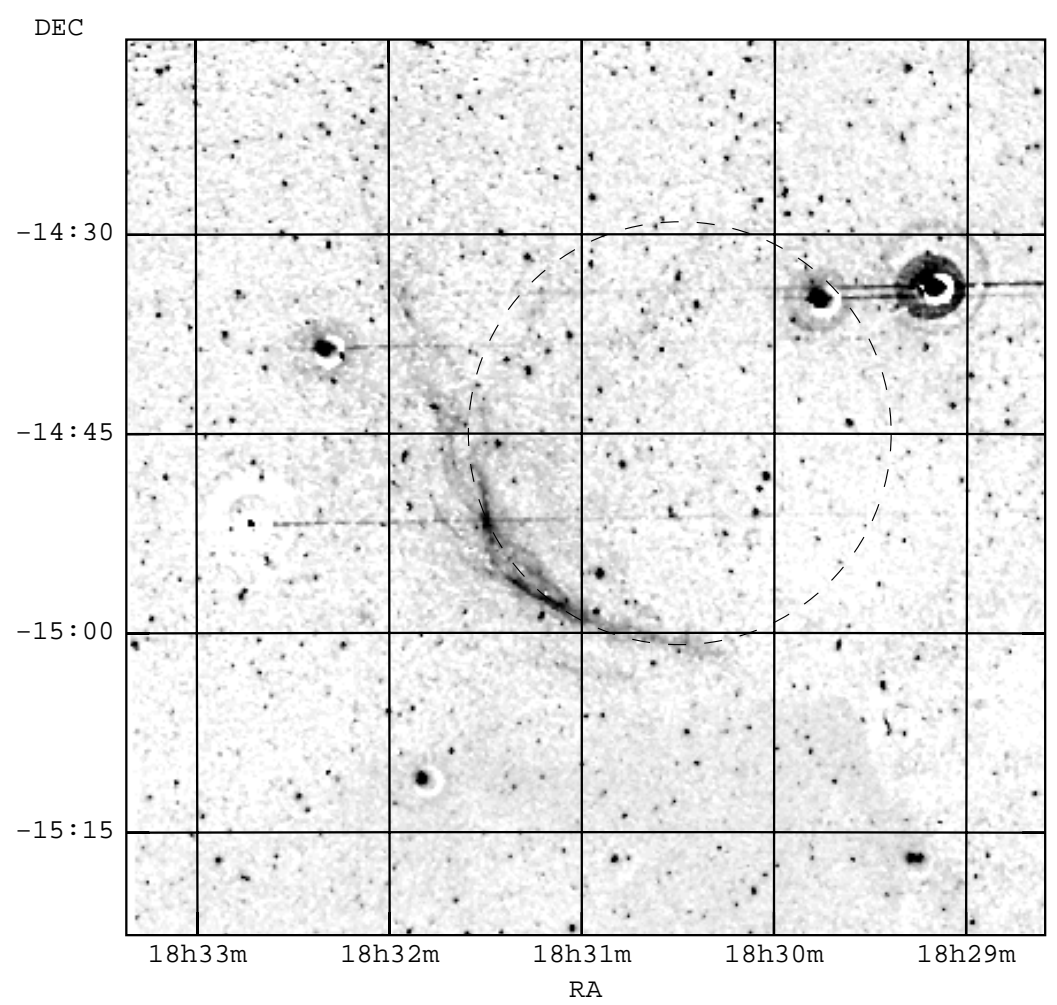

Fig. 3. G 17.4-2.3 imaged with the medium ionization line of [O III $5007 \AA$. The image has been smoothed to suppress the residuals from the imperfect continuum subtraction and the shadings run linearly from 0 to $30 \times 10^{-17} \mathrm{erg} \mathrm{s}^{-1} \mathrm{~cm}^{-2} \operatorname{arcsec}^{-2}$. The projection of a $\sim 26^{\prime}$ diameter sphere that matches the location and orientation of the filament is also shown.

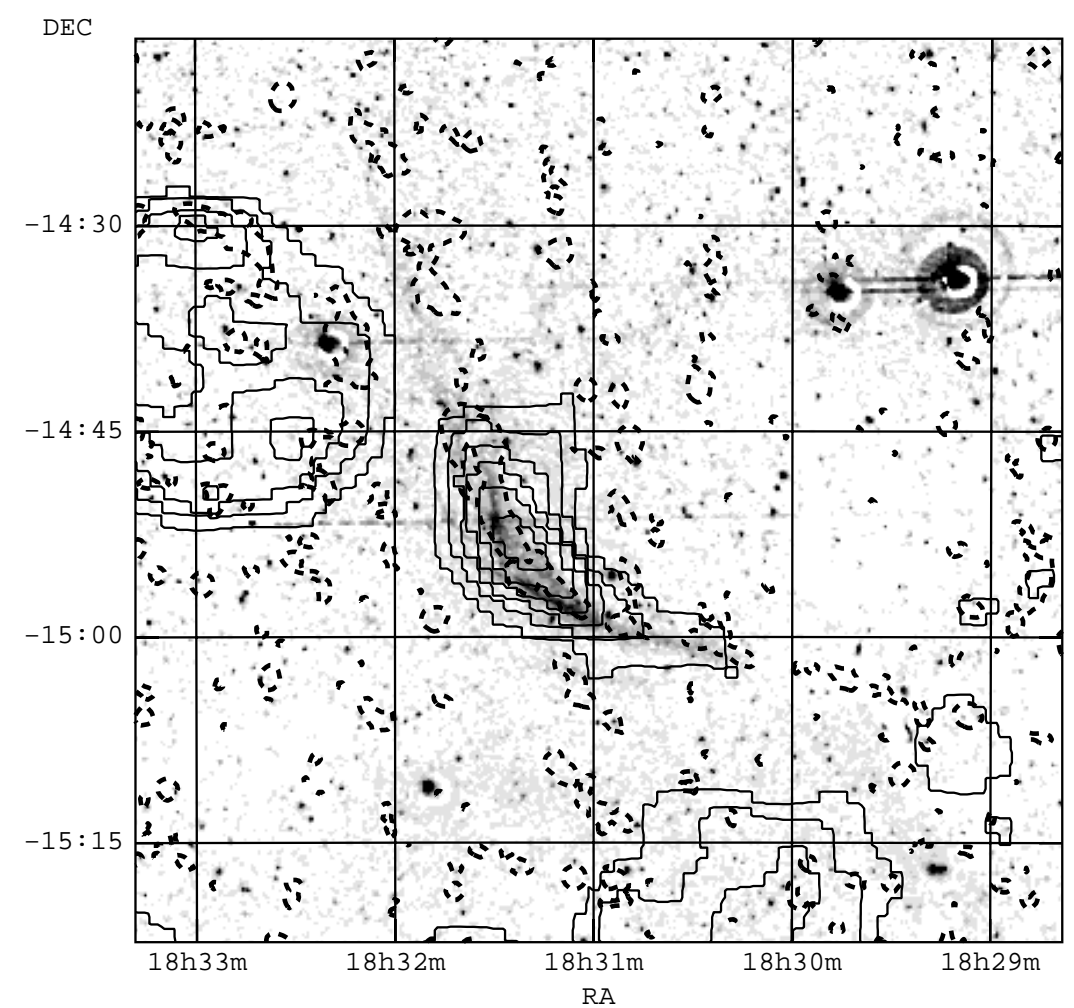

Fig. 4. The correlation between the [O III] emission and the radio emission at $1400 \mathrm{MHz}$ (dash line) and $4850 \mathrm{MHz}$ (solid line) is shown in this figure. The $1400 \mathrm{MHz}$ (Condon et al. 1998) and the $4850 \mathrm{MHz}$ (Condon et al. 1994) radio contours scale linearly from $1.1 \times 10^{-3} \mathrm{Jy} /$ beam to $0.1 \mathrm{Jy} /$ beam and $3.5 \times 10^{-2} \mathrm{Jy} /$ beam to $0.3 \mathrm{Jy} / \mathrm{beam}$, respectively. LDN 379 is also a strong radio source although not detected in [O III]. Note that in the north-east area of the filament we find the well-defined radio shell of the SNR G 17.8-2.6, which is also not detected in the optical. 

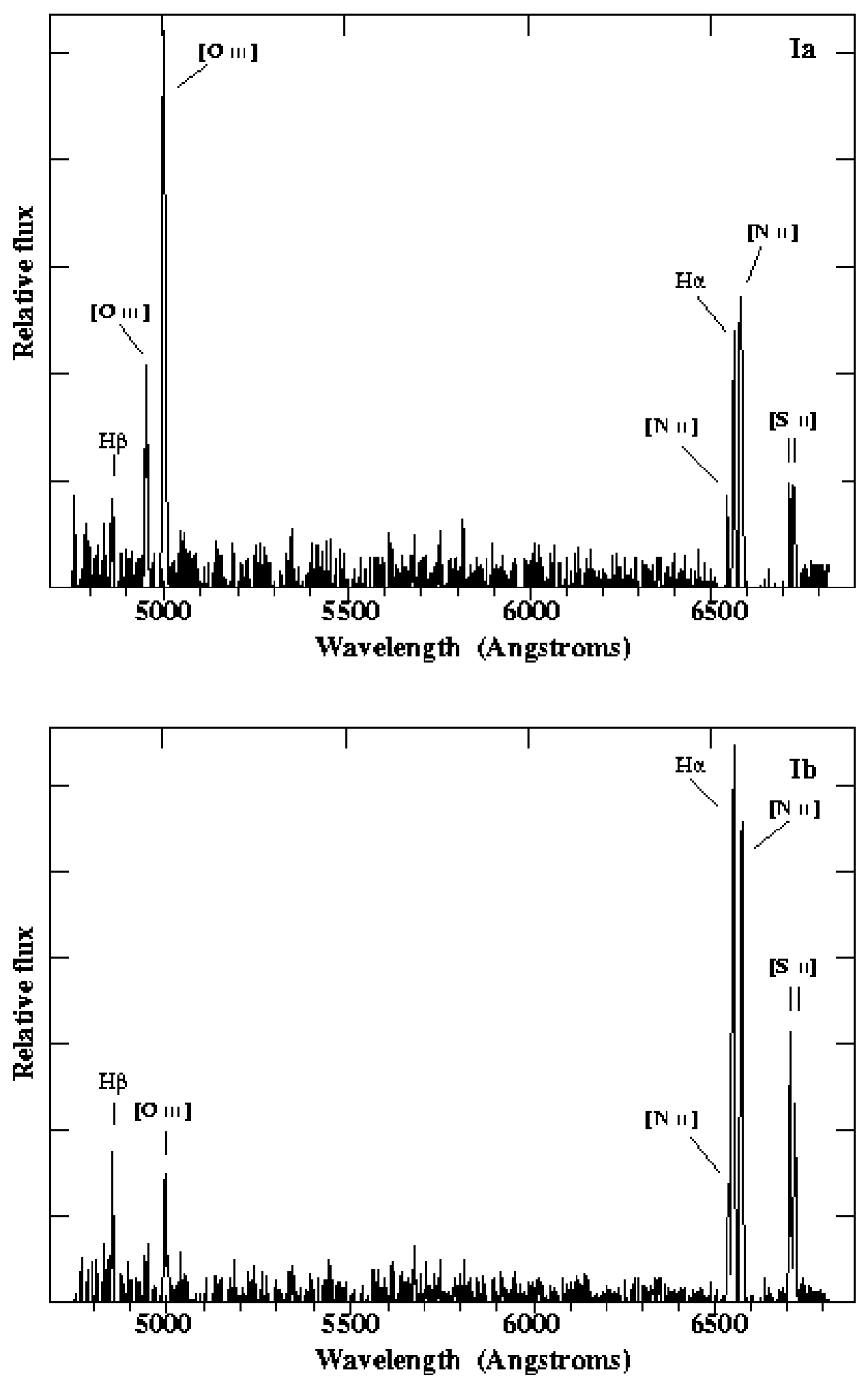

Fig. 5. The spectra of apertures Ia (top) and Ib (bottom).

\section{The long-slit spectra from G 17.4-2.3}

The low resolution spectra were taken on the relatively bright optical filament in the east part of the remnant (its exact position is given in Sect. 2.2). In Table 2, we present the relative line fluxes taken from two different apertures (Ia and Ib, Fig. 5) along the slit. In particu- lar, apertures Ia and Ib have an offset of $9^{\prime \prime} .5$ and $118^{\prime \prime} .5$ north of the slit centre, respectively. The apertures Ia and Ib were selected because they are free of field stars in an otherwise crowded field. In addition, these apertures include sufficient line emission, especially in the blue part of the spectrum, to allow an accurate determination of 
Table 2. Relative line fluxes.

\begin{tabular}{lll}
\hline \hline & Ia & Ib \\
\hline Line $(\AA)$ & $F^{\mathrm{a}, \mathrm{b}}$ & $F^{\mathrm{a}, \mathrm{b}}$ \\
\hline $4861 \mathrm{H} \beta$ & $22(3)^{\mathrm{c}}$ & $15(6)$ \\
\hline $4959[\mathrm{OIII}]$ & $70(9)$ & $4(2)$ \\
\hline $5007[\mathrm{OIII}]$ & $221(28)$ & $23(9)$ \\
\hline $6548[\mathrm{~N} \mathrm{II}]$ & $39(13)$ & $21(16)$ \\
\hline $6563 \mathrm{H} \alpha$ & $100(30)$ & $100(75)$ \\
\hline $6584[\mathrm{~N} \mathrm{II}]$ & $142(44)$ & $75(57)$ \\
\hline $6716[\mathrm{~S} \mathrm{II}]$ & $44(15)$ & $41(34)$ \\
\hline $6731[\mathrm{~S} \mathrm{II}]$ & $38(13)$ & $30(25)$ \\
\hline \hline $\mathrm{Absolute} \mathrm{H} \alpha$ flux $^{\mathrm{d}}$ & 7.9 & 19 \\
\hline $\mathrm{H} \alpha / \mathrm{H} \beta$ & $4.5(3)$ & $6.7(6)$ \\
\hline$[\mathrm{S} \mathrm{II}] / \mathrm{H} \alpha$ & $0.82(17)$ & $0.71(36)$ \\
\hline$F(6716) / F(6731)$ & $1.2(10)$ & $1.4(20)$ \\
\hline
\end{tabular}

${ }^{\text {a }}$ Uncorrected for interstellar extinction.

b Listed fluxes are a signal to noise weighted average of the individual fluxes.

c Numbers in parentheses represent the signal to noise ratio. of the quoted fluxes.

${ }^{\mathrm{d}}$ In units of $10^{-17} \mathrm{erg} \mathrm{s}^{-1} \mathrm{~cm}^{-2} \operatorname{arcsec}^{-2}$.

All fluxes normalized to $F(\mathrm{H} \alpha)=100$.

the observed lines. The background extraction aperture was taken towards the north end of the slit. We utilized the flux calibrated images to identify the nature of this background emission used in the spectra. The $[\mathrm{SII}] / \mathrm{H} \alpha$ ratio of $\sim 0.2$ measured in the images suggests an $\mathrm{H}$ II origin for the background aperture emission. Assuming that its strength does not change appreciably at the Ia and Ib positions we performed the background subtraction to identify the SNR emission. The signal to noise ratios presented in Table 2 do not include calibration errors, which are less than $10 \%$. Both extracted apertures show clearly that the observed optical emission originates shock heated gas, since the $[\mathrm{S} \mathrm{II}] / \mathrm{H} \alpha>0.7$. The $[\mathrm{S} \mathrm{II}] \lambda 6716 / 6731$ ratio of 1.2-1.4 indicates low electron densities (Osterbrock 1989).

The very strong [O III] emission detected in aperture Ia suggests a shock velocity greater than $100 \mathrm{~km} \mathrm{~s}^{-1}$ (Cox \& Raymond 1985), while the sulfur lines ratio indicate an electron density $\sim 240 \mathrm{~cm}^{-3}$ (Osterbrock 1989). However, taking into account the statistical errors on the sulfur lines, electron densities up to $400 \mathrm{~cm}^{-3}$ are compatible with our measurements. The shock velocity implies by the Ib spectrum could be less but still around $100 \mathrm{~km} \mathrm{~s}^{-1}$, while the electron density is even lower than in Ia (less than $120 \mathrm{~cm}^{-3}$ ).

\section{Discussion}

The supernova remnant G 17.4-2.3 shows up as an incomplete shell in the radio bound without any $\mathrm{X}$-ray emission detected so far. The low ionization images generally show diffuse emission in the south and south-east areas of the remnant. In contrast, a filamentary structure has been discovered in the medium ionization [O III] line in the east, south-east region which is very well correlated with the radio emission at 1400 and $4850 \mathrm{MHz}$ and could define the remnant's outer edge. This correlation indicates that the observed emission is associated to G 17.4-2.3. Both the calibrated images and the long-slit spectra suggest that the detected emission results from shock heated gas since the $[\mathrm{S} \mathrm{II}] / \mathrm{H} \alpha$ ratio exceeds the empirical SNR criterion value of $0.4-0.5$. Note that the $\mathrm{H}$ II region found in the low ionization images shows a $[\mathrm{S} \mathrm{II}] / \mathrm{H} \alpha$ ratio of $\sim 0.2$. The eastern filament lies very close to this $\mathrm{H}$ II region. The morphological differences between the low and medium ionization lines provide evidence for significant inhomogeneities and density variations in the ambient medium. Hester et al. (1987) suggested that the presence of such inhomogeneities and density variations would mainly affect the recombination zone where the low ionization lines are produced and it could also explain the $[\mathrm{O}$ III $] / \mathrm{H} \alpha$ ratio variations seen in the long-slit spectra.

An interstellar extinction c at positions Ia and Ib (see Table 2$)$, of $0.62( \pm 0.33)$ and $1.10( \pm 0.17)$ or an $A_{\mathrm{V}}$ of 1.27 $( \pm 0.67)$ and $2.26( \pm 0.35)$ were measured, respectively. We have also determined the electron density measuring the density sensitive line ratio of $[\mathrm{S} \mathrm{II}] \lambda 6716 / 6731$. The densities we measure are below $400 \mathrm{~cm}^{-3}$. Assuming that the temperature is close to $10^{4} \mathrm{~K}$, it is possible to estimate basic SNR parameters. The remnant under investigation is one of the least studied remnants and thus, the current stage of its evolution is unknown. The possibilities that the remnant is still in the adiabatic phase or in the radiative phase of its evolution cannot be excluded and will be examined in the following. The preshock cloud density $n_{\mathrm{c}}$ can be measured by using the relationship (Dopita 1979)

$n_{[\mathrm{SII}]} \simeq 45 n_{\mathrm{c}} V_{\mathrm{s}}^{2} \mathrm{~cm}^{-3}$,

where $n_{[\mathrm{SII}]}$ is the electron density derived from the sulfur line ratio and $V_{\mathrm{s}}$ is the shock velocity into the clouds in units of $100 \mathrm{~km} \mathrm{~s}^{-1}$. Furthermore, the blast wave energy can be expressed in terms of the cloud parameters by using the equation given by McKee \& Cowie (1975)

$E_{51}=2 \times 10^{-5} \beta^{-1} n_{\mathrm{c}} V_{\mathrm{s}}^{2} r_{\mathrm{s}}^{3}$ erg.

The factor $\beta$ is approximately equal to 1 at the blast wave shock, $E_{51}$ is the explosion energy in units of $10^{51} \mathrm{erg}$ and $r_{\mathrm{s}}$ the radius of the remnant in pc. By using the upper limit on the electron density of $400 \mathrm{~cm}^{-3}$, which was derived from our spectra, we obtain from Eq. (1) that $n_{\mathrm{c}} V_{\mathrm{s}}^{2}<8.9$. Then Eq. (2) becomes $E_{51}<0.08 D_{1 \mathrm{kpc}}^{3}$, where $D_{1 \mathrm{kpc}}$ the distance to the remnant in units of $1 \mathrm{kpc}$.

An estimated value of $N_{\mathrm{H}} \sim 5 \times 10^{21} \mathrm{~cm}^{-2}$ is given by Dickey \& Lockman (1990) for the column density in the direction of G 17.4-2.3. Considering that (Ryter et al. 1975)

$N_{H}=6.8( \pm 1.6) \times 10^{21} E_{B-V} \mathrm{~cm}^{-2}$, 
where $A_{\mathrm{V}}=3.1 \times E_{B-V}$ (Kaler 1976), we obtain an $N_{\mathrm{H}}$ of $3 \times 10^{21} \mathrm{~cm}^{-2}$ and $5 \times 10^{21} \mathrm{~cm}^{-2}$ for the two c values calculated from our spectra, respectively. A column density of $2_{-2}^{+1} \times 10^{21} \mathrm{~cm}^{-2}$ was measured towards an $\mathrm{X}$-ray binary system, located $1.2^{\circ}$ east of $\mathrm{G} 17.4-2.3$ at a distance of $\sim 3.1 \mathrm{kpc}$ (Ribó et al. 1999). Since, there are no other measurements of the interstellar density $n_{0}$, values of 0.1 and 1.0 will be examined. Following the result of Eq. (2) and assuming the typical value of 1 for the supernova explosion energy $\left(E_{51}\right)$, we find that the remnant may lie at distances greater than $2.3 \mathrm{kpc}$. Then, the lower interstellar density of $\sim 0.1 \mathrm{~cm}^{-3}$ suggests that the column density is greater than $7 \times 10^{20} \mathrm{~cm}^{-2}$, while for $n_{0} \approx 1 \mathrm{~cm}^{-3}$ it becomes greater than $7 \times 10^{21} \mathrm{~cm}^{-2}$. Combining the previous results and assuming that the column density is found in the range of $2-5 \times 10^{21} \mathrm{~cm}^{-2}$, then the lower interstellar density seems to be more probable.

Using the results of Cioffi et al. (1988) and the above range of parameters for $n_{0}$ of 0.1 and $1 \mathrm{~cm}^{-3}$, the pressure driven snowplow (PDS) radii are calculated as 38 and $14 \mathrm{pc}$, respectively. The radii, when compared with the estimated SNR radius of $17 D_{2.3 \mathrm{kpc}}$ pc may suggest that the PDS phase has not begun yet. The derived velocities of the main shock front at the beginning of this stage are $\sim 300-400 \mathrm{~km} \mathrm{~s}^{-1}$. Their comparison with the estimated shock velocities into the interstellar clouds result in a density contrast of $\sim 10$ between the ambient medium and the interstellar "clouds". However, since neither the distance nor the interstellar medium density are accurately known, we cannot confidently determine the current stage of evolution of G $17.4-2.3$.

\section{Conclusions}

The faint supernova remnant G 17.4-2.3 was observed for the first time in major optical emission lines. The images show filamentary and diffuse emission structures. The bright [O III] filament is very well correlated with the remnant's radio emission at 1400 and $4850 \mathrm{MHz}$ suggesting their association. The flux calibrated images and the longslit spectra indicate that the emission arises from shock heated gas. The observed optical filamentary structure provides some evidence for significant inhomogeneities in the ambient medium, implying that the main blast wave propagates into an inhomogeneous medium.

Acknowledgements. The authors would like to thank the referee for the comments and suggestions which helped to clarify, and enhance, the scope of this paper. Skinakas Observatory is a collaborative project of the University of Crete, the Foundation for Research and Technology-Hellas and the MaxPlanck-Institut für Extraterrestrische Physik. This research has made use of data obtained through the High Energy Astrophysics Science Archive Research Center Online Service, provided bythe NASA/Goddard Space Flight Center.

\section{References}

Case, G. L., \& Bhattacharya, D. 1998, ApJ, 504, 761

Cioffi, D. F., McKee, C. F., \& Bertschinger, E. 1988, ApJ, 334, 252

Condon, J. J., Broderick, J. J., Seielstad, G. A., Douglas, K., \& Gregory, P. C. 1994, AJ, 107, 1829

Condon, J. J., Cotton, W. D., Greisen, E. W., et al. 1998, AJ, 115, 1693

Cox, D. P., \& Raymond, J. C. 1985, ApJ, 298, 651

Dickey, J. M., \& Lockman, F. J. 1990, ARA\&A, 28, 215

Dopita, M. A. 1979, ApJS, 40, 455

Fesen, R. A., Blair, W. P., \& Kirshner, R. P. 1985, ApJ, 292, 29

Fesen, R. A., \& Hurford, A. P. 1995, AJ, 110, 747

Fesen, R. A., Winkler, P. F., Rathore, Y., et al. 1997, AJ, 113, 767

Green, D. A., Frail, D. A., Goss, W. M., \& Otrupcek, R. 1997, AJ, 114, 2058

Green, D. A. 2000, A Catalog of Galactic Supernov a Remnants (2000 August version), Mullard Radio Astronomy Observatory (Cambridge)

Hester, J. J. 1987, ApJ, 314, 187

Hilton, J., White, G. J., Cronin, N. J., \& Rainey, R. 1986, A\&A, 154, 274

Hamuy, M., Walker, A. R., Suntzeff, N. B., et al. 1992, PASP, 104,533

Hamuy, M., Suntzeff, N. B., Heathcote, S. R., et al. 1994, PASP, 106, 566

Kaler, J. B. 1976, ApJS, 31, 517

Lasker, B. M., Russel, J. N., \& Jenkner, H. 1999, in the HST Guide Star Catalog, version 1.1-ACT, The Association of Universities for Research in Astronomy, Inc

Lynds, B. T. 1965, ApJS, 12, 163

Mavromatakis, F., Papamastorakis, J., Paleologou, E. V., \& Ventura, J 2000, A\&A, 353, 371

Mavromatakis, F., Papamastorakis, J., Ventura, J., et al. 2001, A\&A, 370, 265

Mavromatakis, F., Boumis, P., \& Paleologou, E. V. 2002, A\&A, 383,1011

McKee, C. F., \& Cowie, L. L. 1975, ApJ, 195, 715

Osterbrock, D. E. 1989, Astrophysics of gaseous nebulae (W. H. Freeman \& Company)

Raymond, J. C. 1979, ApJS, 39, 1

Reich, W., Fürst, E., Reich, P., \& Junkes, N. 1988, in IAU Colloq. 101, Supernova Remnants and the Interstellar Medium, ed. Roger R. S., \& Landecker T. L. (Cambridge Univ. Press), 293

Reich, W., Fürst, E., Reich, P., \& Reif, K. 1990, A\&AS, 85, 633

Ribó, M., Reig, P., Martí, \& Paredes, J. M. 1999, A\&A, 347, 518

Ryter, C., Cesarsky, C. J., \& Audouze, J. 1975, ApJ, 198, 103

Seward, F. D., Dame, T. M., Fesen, R. A., \& Aschenbach, B. 1995, ApJ, 449, 681

Shull, J. M., \& McKee, C. F. 1979, ApJ, 227, 131

Smith, R. C., Kirshner, R. P., Blair, W. P., Long, K. S., \& Winkler, P. F. 1993, ApJ, 407, 564 